\title{
BIOLOGICAL METHOD FOR BABESIOSIS DETECTION: THE UNIFIED VERSION IN VIVO
}

D0I:10.36740/WLek202102117

\author{
Inna I. Torianyk \\ STATE INSTITUTION «MECHNIKOV INSTITUTE OF MICROBIOLOGY AND IMMUNOLOGY OF THE NATIONAL ACADEMY OF MEDICAL SCIENCES OF \\ UKRAINE», KHARKIV, UKRAINE
}

\begin{abstract}
The aim is to establish a unified version of the biological method for babesiosis detection in vivo.

Materials and methods: samples ( $n=257$ ) of biological material of different origin were examined. These included: blood samples from patients $(n=6)$ and cattle $(n=15)$; salivary gland homogenates $(n=28)$ from 147 imagoes of ticks of the family lxodidae, 32 imagoes of Ixodes ricinus and 115 imagoes of Dermacentor reticulates; spleen homogenates $(n=63)$ from mouse-like rodents (Muridae) of the genera Myodes, Microtus, Apodemus and Sylvaemus. In order to cultivate in vivo Babesiae of the species B. microti, Syrian hamsters were infected with spleen homogenates from mouse-like rodents; for cultivating the B. divergens species Mongolian gerbils and nonlinear white mices were infected with blood samples from patients and cattle and salivary gland homogenates from ixodic ticks. The technology of modeling was based on the group specificity (differences in susceptibility to parasites and in parameters of morbidity) of the animals, involved in the experiment (Syrian hamsters, Mongolian gerbils, nonlinear white mices).

Results: Experimental animals were contaminated by means of intraperitoneal inoculation of $0.3 \mathrm{ml}$ samples of biological material (infected with Babesiae). The animals were infected next day following a day of their preinoculation preparation. The marker parameters for the functional state of experimental animals were as follows: preterm death; appearance and development of clinical-laboratory signs of disease (hypo- or adynamia, loss of appetite, inertness/absence of reactogenicity to tactile/acoustical stimulation, postural changes, wetting of fur, pronounced lameness, flatulence, loss of $\geq 25 \%$ of body mass) in them; parasitaemia, histodestruction, cellular detritis. Parasitaemia was detected every two days (beginning with day 8 from the moment of inoculation) by reserves of light and luminescent microscopy. In case of the positive result (revealing of haemoparasites with Babesia spp.-like morphological and tinctorial signs) the verification of Babesiae with their more precise specific identification was performed using the technique of polymerase chain reaction (PCR). Preliminary detection of morbidity parameters in each experimental animal with the artificially created immunocompromised state became an obligatory moment of the described experiment.

Conclusions: The biological method for detecting Babesia spp. in vivo was improved by the author. This result was achieved by using a double reservoir (Syrian hamsters, Mongolian gerbils and nonlinear white mices with an increased level of susceptibility to parasites) followed by the immunocompromise formation. The use of the improved version of biological method increased the total rate of revealing of Babesiae, therewith creating an objective basis for optimizing the available ways of detection and study of Babesiae in vivo.
\end{abstract}

KEY WORDS: biological method, detection, Syrian hamsters, Mongolian gerbils, nonlinear white mices, babesiosis

\section{INTRODUCTION}

Babesiosis as a little-studied emergent haemoparasitic infection presents certain difficulties in its detection and differential diagnosis [1-3]. Species specificity of the causative agents of the above disease determines the conjuncture of both etiopathogenetic scenario of babesiosis and its numerous clinical episodes, the catamnetic plot [4-6]. A full understanding of the latter demands that researchers (including in the sense of creating a rich diagnostic evidential base) both use modern novel ways and orient themselves on (apply) a time-tested classic of the genre (in which experimental modeling takes not the last place). An expected contribution to solving the above problem is made by combined diagnostics. Hence, designing and development of improved clinical-diagnostic algorithms of babesiosis are an urgent problem of both theoretical and fundamental fields of medicine.

\section{THE AIM}

The aim is to establish a unified version of the biological method for babesiosis detection in vivo.

\section{MATERIALS AND METHODS}

In order to reveal and cultivate the causative agents of babesiosis (Babesia spp.) we studied samples $(n=257)$ of biological material of different origin. These included: blood samples from patients, who lived in the Kharkiv Region, with diagnosed Lyme disease resulting from a tick bite $(n=6)$; cattle $(n=15)$ with suspected Babesiae invasion (the Chernihiv and Poltava Regions, Ukraine). The sample scope also included salivary gland homogenates $(n=28)$ from 147 imagoes of ticks of the family Ixodidae, 32 imagoes of Ixodes ricinus and 115 imagoes of Dermacentor reticulates, taken from resources of field studies 


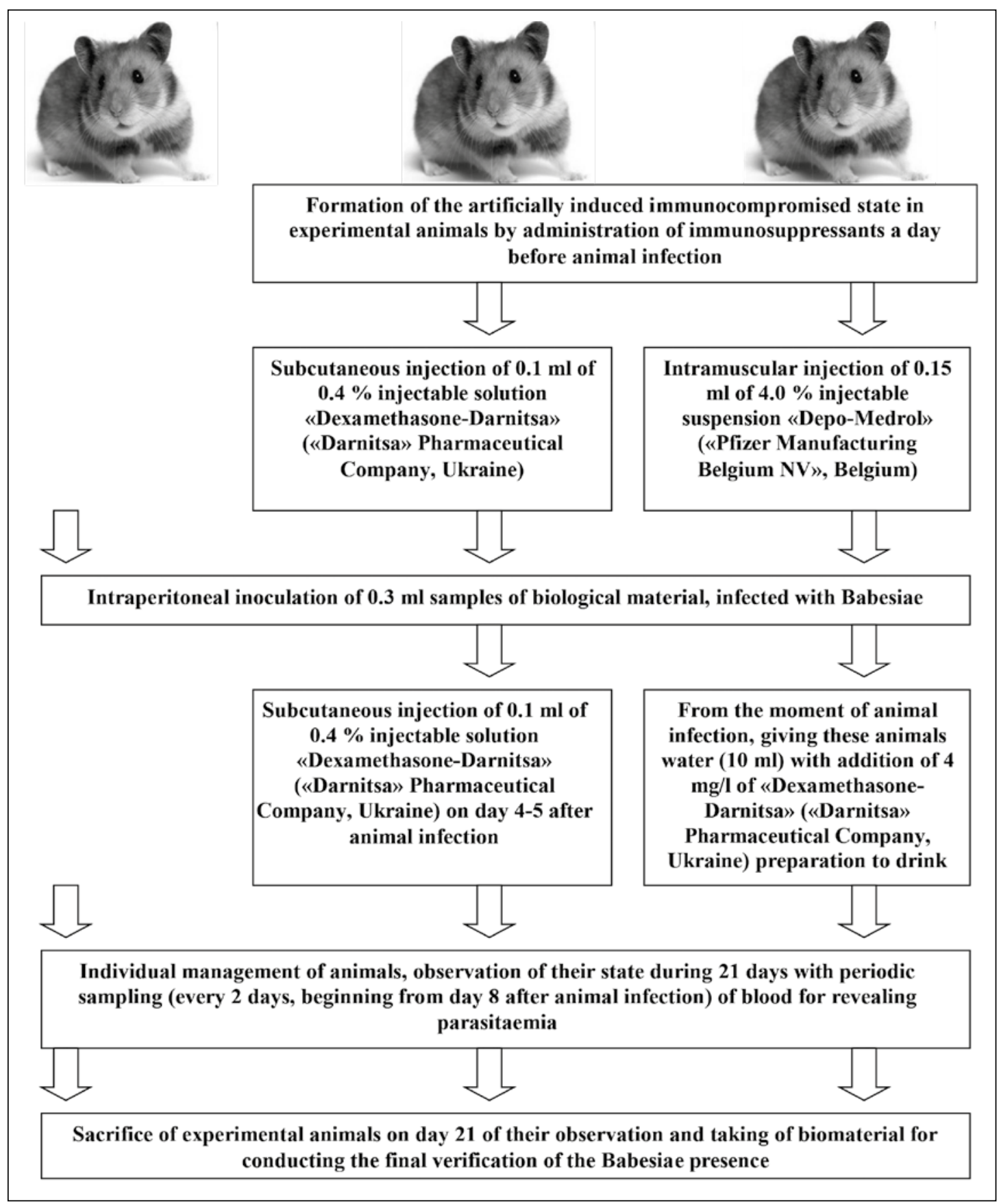

Fig. 1. The algorithm of detection and cultivation of Babesiae in vivo with use of Syrian hamsters.

in epizootic areas of the Kharkiv, Volyn, Chernihiv and Zhytomyr Regions (Ukraine). Spleen homogenates $(n=63)$ from mouse-like rodents (Muridae) of the genera Myodes, Microtus, Apodemus and Sylvaemus, caught in natural ecotopes of the Zhytomyr, Poltava and Kharkiv Regions (Ukraine), were an obligatory object of the described study.

Detection and in vivo cultivation of Babesiae (the species B. microti) were made on males and females of Syrian hamsters (Mesocricetus auratus, $\mathrm{n}=45$ ) with body mass of 30-45 $\mathrm{g}$ and at the age of 9-22 weeks [7-11]. Proper experimental procedures with the species $B$. divergens were performed on males and females of Mongolian gerbils (Meriones unguiculatus, $n=55$ ) with body mass of 45-90 $\mathrm{g}$ and at the age of 9-22 weeks and nonlinear white mices $(n=45)$ with body mass of 11-18 $\mathrm{g}$ and at the age of 4-6 weeks [6-11].

The laboratory animals with proper documents, presented in the appropriate registration form, from the veterinary service of Ukraine were placed under quarantine and managed in standard conditions («climate-control») at the vivarium of the State Institution «Mechnikov Institute of Microbiology and Immunology of the National Academy of Medical Sciences of Ukraine».

Air temperature was $18-24^{\circ} \mathrm{C}$, taking into account the poikilothermy of each animal; relative humidity did not exceed the level of 50-70\%; artificial illumination was normally maintained with the value of $60 \mathrm{~lx}$ with a 12 - 


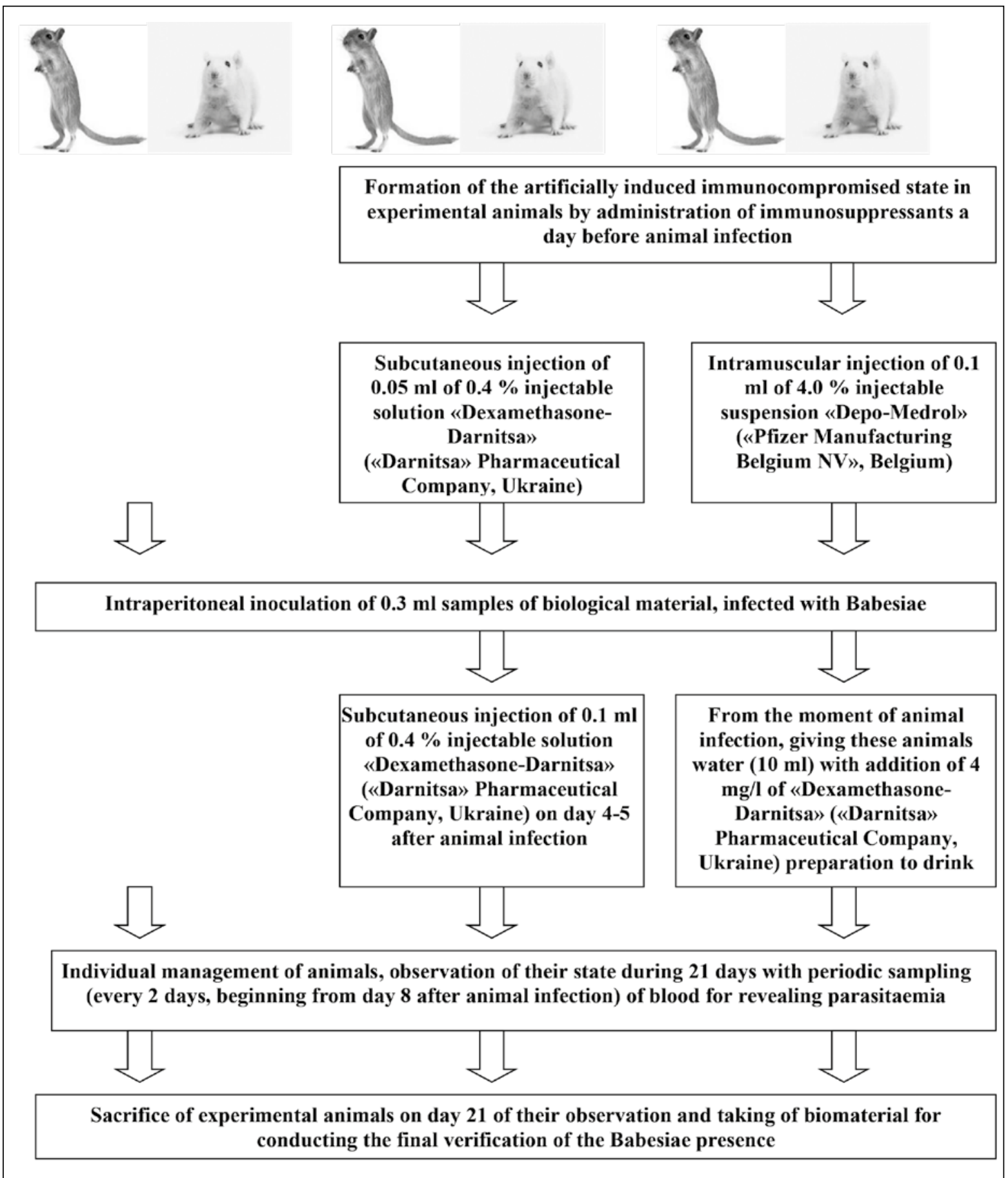

Fig. 2. The algorithm of detection and cultivation of Babesiae in vivo with use of Mongolian gerbils and nonlinear white mices.

hour cycle. Manipulations with selection, preparation and studying of biological material samples were performed with observation of rules of aseptics for the maximally possible prevention of their additional contamination with nonstudy microflora $[4,5]$ at operating rooms of the vivarium of the State Institution «Mechnikov Institute of Microbiology and Immunology of the National Academy of Medical Sciences of Ukraine» with a direct participation of a veterinary doctor in morning hours. The latter fact was caused by biorhythmical activity and chronobiological features of laboratory animals [1]. From the morning of the day of the experimental intervention any feeding of the animals was excluded. During the whole nonexperimental period the feeding of the animals was organized in strict compliance with developed scientific standards, was of the rational and balanced nature, and conformed with daily requirements of feeding stuffs (with calculations considering weight, age and sex indices of each animal). Access to water and food remained free. Standard extruded foodstuff and water were given in sufficient quantity (in the amount of 30-32 g per each animal in order to avoid cases of cannibalism) two times a day (in the postexperimental period ad libitum) with periodic analysis of foodstuff samples for microbiological contamination [1]. Taking into 
consideration that the rodents, used in the experiment, were nocturnal animals and ate in hours of darkness too, most of their foodstuff was given in the evening, at 8 p.m. [3]. All animals were examined every day by a veterinary doctor, who exercised control over the animals' vital activity during the whole experiment (postprocedure/ manipulation period, sampling of biological material, removal from the experiment) [7]. Sacrifice was made by hyperanaesthetization with chloroform. The conditions of animal management and care satisfied requirements of international and national documents.

\section{RESULTS AND DISCUSSION}

The biological method of babesiosis diagnosing in humans is to detect Babesia spp. by growing them in vivo in the body of sensitive experimental animals [1-4].

In order to cultivate in vivo Babesiae of the species B. microti, Syrian hamsters were infected with spleen homogenates from mouse-like rodents (fig. 1). In order to detect and cultivate in vivo Babesiae of the species B. divergens, Mongolian gerbils and nonlinear white mices were infected with blood samples from patients with Lyme disease, from cattle with Babesiae invasion and with salivary gland homogenates from ixodic ticks (fig. 2).

In order to objectify elements of reproduction of biological method, detection and cultivation of Babesia spp. we carried out preinoculation preparation of laboratory animals for formation of the induced (acquired) immunocompromised state in them. The latter facilitated in improving susceptibility of experimental animals to parasites and provided conditions for intensive growth of the above parasites in the infected organism and formation of morbidity.

The technology of modeling was based on the group $(n=3)$ specificity (differences in susceptibility to parasites and in parameters of morbidity) of the animals, involved in the experiment (Syrian hamsters, Mongolian gerbils, nonlinear white mices).

The control group (G 1) consisted of 103 animals (31 Syrian hamsters, 41 Mongolian gerbils, 31 nonlinear white mices), who were infected with biomaterial samples without any preinoculation preparation. The immunocompromised state in the above animals was absent.

The second group (G 2) was made up 21 animals (by 7 animals in each subgroup of Syrian hamsters, Mongolian gerbils, nonlinear white mices). The above animals were characterized by an artificially created immunocompromised state. The latter was formed by subcutaneous injections of $0.05 \mathrm{ml}$ (for Mongolian gerbils and nonlinear white mices) or $0.1 \mathrm{ml}$ (for Syrian hamsters) of $0.4 \%$ injectable solution «Dexamethasone-Darnitsa» («Darnitsa» Pharmaceutical Company, Ukraine). The experimental animals were contaminated by means of intraperitoneal inoculations of $0.3 \mathrm{ml}$ samples of biological material (infected with Babesiae). The experimental specificity of the test animals from $\mathrm{G} 2$ consisted in the fact that on days 4-5 after their contamination with biomaterial samples they were given another injection of $0.4 \%$ injectable solution «Dexamethasone-Darnitsa» («Darnitsa» Pharmaceutical Company, Ukraine).

The third group (G3) included 21 animals (by 7 animals in each subgroup of Syrian hamsters, Mongolian gerbils, nonlinear white mices). The artificially induced immunocompromised state was achieved by intramuscular injections of $0.1 \mathrm{ml}$ (for Mongolian gerbils and nonlinear white mices) or $0.15 \mathrm{ml}$ (for Syrian hamsters) of $4.0 \%$ injectable suspension «Depo-Medrol» («Pfizer Manufacturing Belgium NV», Belgium). After that, the animals were infected according to the developed scheme. Peculiar for the above experimental group were manipulations with giving these infected animals water $(10 \mathrm{ml})$ with addition of $4 \mathrm{mg} / \mathrm{l}$ of «Dexamethasone-Darnitsa» («Darnitsa» Pharmaceutical Company, Ukraine) preparation to drink.

Preliminary detection of morbidity parameters of each animal with the artificially formed immunocompromised state was an obligatory component of the described experiment. The last manipulation was targeted at revealing a potential presence of the causative agents (wild strains) in the organism of experimental animals involved in the study. By the results of our observations (21 days) the whole sample scope of noninfected imunnocompromised animals went out of the test experiment without any cases of lethality, disease and asymptomatic parasitaemia. It is this contingent of animals that was engaged in further experiments with biological method (in vivo) for detecting and cultivating Babesia spp.

The marker parameters for the functional state of experimental animals of G 2 and G 3 were as follows: preterm death; formation and development of clinical-laboratory signs of disease (hypo- or adynamia, loss of appetite, inertness/absence of reactogenicity to tactile/acoustical stimulation, postural changes, wetting of fur, pronounced lameness, flatulence, loss of $\geq 25 \%$ of body mass); parasitaemia, histodestruction (marked inflammatory reactions, destruction of title cells of marker organs, necrosis), large accumulation of cellular detritis. Detection of parasitaemia (search for intra- and extraerythrocytic inclusions) was performed every two days (beginning from the moment of inoculation) by reserves of light and luminescent microscopy. In case of the positive result (revealing of haemoparasites with Babesia spp.-like morphological and tinctorial signs) the verification of Babesiae with their more precise specific identification was performed using the technique of PCR. The latter was carried out in its standard format using commercial test systems (manufactured by «IsoGene Lab. Ltd», the Russian Federation): the kit of reagents for amplifying deoxyribonucleic acid (DNA) of Babesia spp. «Gene Pak ${ }^{\circledR} \mathrm{P}$ CR test» (with a system of Bab primers), Cat. No. E 2130, the kit of reagents for amplifying DNA of Babesia microti "Gene Pak ${ }^{\circledast}$ PCR test» (with a system of Bmi primers), Cat. No. B 2129 and the kit of reagents for amplifying DNA of Babesia divergens "Gene Pak ${ }^{\circledR} \mathrm{PCR}$ test» (with a system of Bdi primers), Cat. No. E 2126, respectively.

The animals, which died before their time or were sacrificed (the final terms of the experiment), underwent an 
obligatory autopsy. The latter was performed in conditions of a veterinary dissection room with a thorough somato-organoscopic analysis, detailed revision of abdominal organs, revealing of topographic specificity, holo-, skeleto-, syn- and vasotopy. The above was followed by taking of the organs, targeted for babesiosis (spleen, liver, stomach and small intestine; as for the last two, with consideration of the response of their lymphoid structures). Blood was received by the puncture method of heart or lingual artery (while the animals were alive). Blood samples were used for making model test specimens in the context of immunological studies of consequences of Babesiae invasion for detecting dominant species. Fragments of targeted organs were kept in RPMI 1640 (Sigma-Aldrich, USA) medium at $\mathrm{t}^{\mathrm{o}}=-32^{\circ} \mathrm{C}$ for receiving cultures.

\section{CONCLUSIONS}

The biological method for detecting Babesia spp. in vivo was improved by the author. This result was achieved by using a double reservoir (Syrian hamsters, Mongolian gerbils and nonlinear white mices with an increased level of susceptibility to parasites) followed by the immunocompromise formation. The use of the improved version of biological method increased the total rate of revealing of Babesiae (up to 35.7\%), therewith creating an objective basis for optimizing available ways of detection, cultivation and further in vivo investigation of Babesiae as a group of little-studied causative agents of tick-borne infections.

\section{REFERENCES}

1. Solano-Gallego L., Sainz Á., Roura X. et al. A review of canine babesiosis: the European perspective. Parasites Vectors. 2016;9(336). https://doi. org/10.1186/s13071-016-1596-0

2. Gray J.S., Estrada-Peña A., Zintl A. Vectors of Babesiosis. Annual Review of Entomology. 2019;64(1):149-165.

3. Krause P.J. Human babesiosis. International Journal for Parasitology. 2019; 49(2):165-174.

4. Zelya O.P., Kukina I.V. Babezioz cheloveka. Human babesiosis. Medical News of North Caucasus. 2020;15(3):449-455. (Ru)

5. Kletsova E.A., Spitzer E.D., Fries B.C., Marcos L.A. Babesiosis in Long Island: review of 62 cases focusing on treatment with azithromycin and atovaquone. Annals of Clinical Microbiology and Antimicrobials. 2017;16(26). https://doi.org/10.1186/s12941-017-0198-9.
6. Parija S.C., Kp D., Venugopal H. Diagnosis and management of human babesiosis. Tropical Parasitology.2015;5(2):88-93.

7. Herwaldt B.L., Cacciò S., Gherlinzoni F. et al. Molecular characterization of a non-Babesia divergens organism causing zoonotic Babesiosis in Europe. Emerging Infectious Diseases. 2003;9(8):943-948.

8. Gray J.S. Identity of the causal agents of human babesiosis in Europe. International Journal of Medical Microbiology. 2006;296(40):131-136.

9. Vannier E., Gewurz B.E., Krause P.J. Human babesiosis. Infectious Disease Clinics of North America. 2008;22(3):469-488.

10. Vannier E., Krause P.J. Update on Babesiosis. Interdisciplinary Perspectives on Infectious Diseases. 2009;2009. https://doi. org/10.1155/2009/984568

11. Yabsley M., Shock B.C. Natural history of zoonotic babesia: role of wildlife reservoirs. International Journal for Parasitology: Parasites and Wild life. 2012;2:18-31.

\section{Acknowledgments:}

The Author of the study sincerely appreciates the comprehensive professional support and precious pieces of advice from Nila A. Chygyrynska.

\section{ORCID and contributionship:}

Inna I. Torianyk: 0000-0001-6843-8808 A,B,C,D,E,F

\section{Conflict of interest:}

The Author declare no conflict of interest.

\section{CORRESPONDING AUTHOR Inna I. Torianyk \\ Laboratory of Viral Infections, State Institution «Mechnikov Institute of Microbiology and Immunology of the National Academy of Medical Sciences of Ukraine» 39 Kamysheva Ivana str., apt. 9, 61038, Kharkiv, Ukraine e-mail: patholognew@ukr.net}

Received: 06.09 .2020

Accepted:08.01.2021
A - Work concept and design, B - Data collection and analysis, C - Responsibility for statistical analysis, $\mathbf{D}$-Writing the article, $\mathbf{E}$-Critical review, $\mathbf{F}$ - Final approval of the article 